\title{
Digital Quadrature Mixing of Lowpass Sigma-Delta Modulators for Switch-mode Power Amplifiers
}

\author{
Keith Finnerty, John Dooley and Ronan Farrell \\ CTVR - The Telecommunications Research Centre, Callan Institute, National University of Ireland Maynooth, Co. Kildare, \\ Ireland
}

\begin{abstract}
In this paper a phase compensation technique for the digital upconversion of a quadrature signal for amplification with switch mode power amplifiers is proposed. When a digital signal generator is used to generate the complex envelope signal care must be taken to compensate for the phase skew between the two paths. If phase compensation is not implemented an image caused by up converting the complex envelope of the modulation signal is created. By compensating for phase skew between the I and $Q$ signal paths it is possible to remove this image signal and enable the transmission of multi carrier signals. As a direct result of this technique there is a reduction in the filtering effort at the output of the power amplifier to meet spectral mask requirements.
\end{abstract}

Keywords-component; power amplifiers, delta-sigma converters, class $S$

\section{INTRODUCTION}

Power amplifiers (PA) are a key component in radio communications systems amplifying the low power radio signals for transmission. This operation consumes a large amount of power and as such PA's are one of the largest consumers of power within a radio system, making the efficiency of a PA important in terms of overall system performance. Switch-mode PA's have the highest theoretical efficiency but require a digital bit stream as an input at RF frequencies. Switch mode power amplifiers have a theoretical efficiency of $100 \%$ assuming ideal switching can be achieved. In practice this is not possible as yet at high frequencies. For audio applications however efficiencies of $85-90 \%$ [1] have been realized. In these low frequency applications amplifiers such as Class-D amplifiers are widely used today. Since the class D amplifier eliminates the envelope of the input signal, all amplitude information from the modulation signal must be encoded in the timing of the switch transitions.

Class-S power amplifiers are a combination of a switch mode power amplifier (Class-D), driven by a sigma delta modulator. The sigma delta modulator (SDM) provides a Pulse Density Modulated (PDM) signal at radio frequency (RF) to drive the switch mode power amplifier. After the amplification stage a bandpass filter is used to remove the quantization noise and this transforms the signal from digital PDM to analogue continuous time signal for transmission.

This paper proposes the method of digital quadrature mixing for up conversion of the signal to an RF transmit frequency using commercially available FPGAs. This can be achieved in two separate ways: (i) using a high pass SDM [2-3] (ii) Quadrature mixing which attenuates the signal image caused by converting a low-if signal to a transmit frequency. The former will place a signal about half the sample frequency (Fs/2) and have an image close to the transmit signal. The latter will increase the available bandwidth of the SDM and removes the need for aggressive filtering to remove the image after amplification. Filtering the image out after amplification is impractical as is requires a high $Q$ filter which can handle the large amounts of power contained within the amplified signal. Image cancellation before amplification will also increase the coding efficiency of the modulator which should in turn improve amplifier efficiency as the amplifier will not be amplifying the unwanted signal image.

\section{SIGMA DELTA MODULATORS FOR CLASS-S}

Sigma delta modulators (SDM) operate by taking a high resolution input and converting it to a two level PDM bit stream. Because of the reduction in resolution to the input signal there is a large amount of quantization noise. This noise is pushed out of the wanted signal band and spread across the frequency spectrum. The shape of the spectral output of the SDM characterizes the system (Low pass, Bandpass or High pass). The characteristic shape is called the noise transfer function. The noise transfer function of a SDM is defined by the number of poles in the loop filter $(\mathrm{H}(\mathrm{z}))$ and their placement.

Previous implementations of Class-S PA's have used direct bandpass SDM's with a PA switching stage. ASIC designs have been used to generate the signals but are not reconfigurable [4]. Designing a SDM stage on an FPGA will allow the system to be reconfigurable while outputting the required PDM signal to drive the PA. FPGA's cannot generate high frequency pulsed signals as well as dedicated CMOS pulse modulators can. Although this is an important consideration when choosing the modulator, FPGAs can provide a good indication of what is possible with further dedicated design of the FPGA high frequency I/Os. The signal produced by the SDM is directly related to the efficiency of the Class-S PA. The signal to be transmitted must be at the transmit frequency and the NTF must have a low noise floor. Many class-S designs use a band pass SDM as it provides a clean signal and low out of band noise, however this requires the SDM frequency to be four times the transmit frequency and can be difficult to design on an FPGA platform. The system in Figure 1 is a quadrature mixing system using two low pass SDMs, these modulators have their notch in the noise transfer function at baseband, with the quantization noise pushed up to the higher frequencies. 
When a signal is digitally mixed from Low-IF up to a carrier frequency an image of the signal is created. The position of the signal will depend on the position of the carrier signal and the Low-IF of the signal before up conversion. The image of the signal is undesirable as it has to be filtered before the signal can be transmitted. Quadrature mixing is a method which cancels the unwanted image caused by up converting a signal from Low-IF.

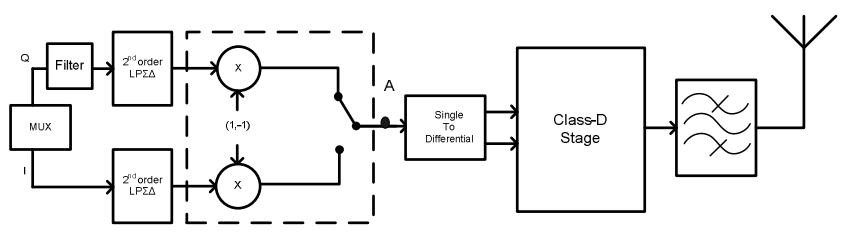

Figure 1 System overview

Quadrature mixing combines the two components of a complex signal, I and Q, which are at Low IF and modulate an $\mathrm{RF}$ carrier. By doing this a wanted signal and image signal will be created about the RF carrier. The Q component of the signal has both the desired signal and the image in phase in the real frequency domain. The I component has the desired signal and image out of phase by $180^{\circ}$ in the imaginary axis frequency domain as demonstrated in Figure 2. During the up conversion a $90^{\circ}$ phase shift is applied to the I component relative to the $\mathrm{Q}$ component, this is achieved by multiplying I component by a sin wave and the $\mathrm{Q}$ component by a cosine wave. This brings I and Q components of the desired signals into phase and they add to amplify the signal. The imaginary components are now $180^{\circ}$ out of phase which means that they cancel. The cancelation can be seen in Figure 2. The quadrature mixing is implemented after the sigma delta stage which is carried out on each of the channels (I and Q). It is important to keep the phase and amplitude of the signal constant as slight changes will affect the image cancellation.

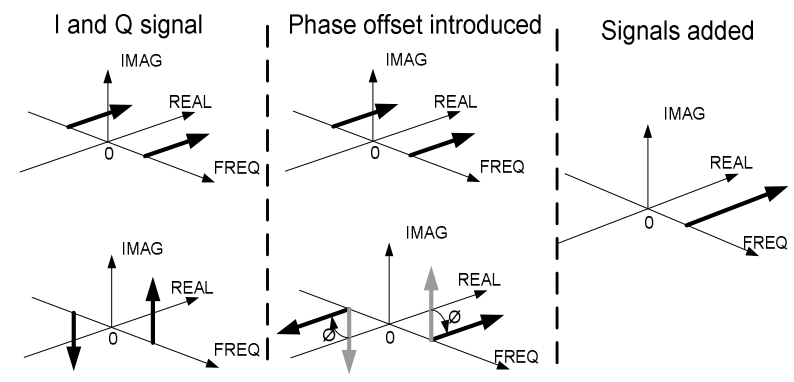

Figure 2 Ideal quadrature mixing

The switch mode PA requires a binary bit stream at an RF carrier frequency. It is possible to generate high frequency bit streams using commercially available FPGA development boards however when the complex modulation signal is digitally up converted an unwanted image is generated close to the wanted RF signal. In order to remove the unwanted image we cancel the imaginary part by phase compensated digital mixing. An overview of the system can be seen in Figure 1. This shows the coarse method for implementing quadrature mixing after the SDM. The signal is up converted using Manchester encoding or 'signal chopping', this transports the desired signal from baseband to half the operating frequency of the SDM $\left(\mathrm{F}_{\mathrm{DSM}}\right)$. The combination of I and Q components and phase shift is performed by the time interleaving process. This method preserves the bits stream in the 1, -1 from that is required to drive the switch mode $\mathrm{PA}$, however due to the time interleaving the signal is now at twice the sample frequency of the original input signal, this up converts the signal from baseband to one quarter the systems sample frequency $(\mathrm{Fs}=$ $\left.2 * \mathrm{~F}_{\mathrm{DSM}}\right)$.

The phase that is introduced to a signal converted from baseband to Fs/4 is $90^{\circ}$ due to the fact that the time interleaving, point A Figure 1, introduces a unit sample delay in a signal. However the phase introduced by interleaving changes as the signal moves away from base band. The phase shift is dependent on the Low-IF frequency of the baseband signal, as the Low-If signal moves from baseband the phase shift introduced by digital quadrature mixing will decrease. If the phase introduced by time interleaving is different than $90^{\circ}$ the quadrature mixing will be affected resulting in an image at twice the Low-IF frequency from the wanted signal. Equation 1 calculates the phase offset incurred from quadrature mixing a Low-IF signal.

$$
\frac{2 \pi}{\frac{F S}{L o w I F}}=\emptyset
$$

An example of the resulting image from the phase imbalance can be seen in Figure 4. The image can have enough power to appear above the NTF and reduce the overall bandwidth within the notch of the NTF. To improve the image cancellation to a point where the image no longer appears above the NTF of the sigma delta a phase offset $\varnothing$ must be introduced to one of the signal paths before the SDM.

\section{QUADRATURE MIXING WITH PHASE CORRECTION}

If the phase offset between two signal paths is smaller than $90^{\circ}$ it cannot be corrected using an integer sample delay so a fractional delay must be used. As explained in the design methodology for fractional delay filters outlined in [4], the Lagrange filter can be designed to act as an all pass fractional delay filter. Figure 3 outlines a Farrow structure which can be used to implement the relative fractional delay required in this application [5], this structure will be placed in the Q path of the system before the sigma delta modulator process. It uses two or more FIR filters in parallel; the outputs are weighted by a factor D which corresponds to the delay that the filter will implement. The coefficients of the filter are $(-1,1)$ for FIR1 and $(1,0)$ for FIR 2.

$$
Y(t)=X(t)+D *\{X(t-1)-X(t)\}
$$


Equation 2 describes the function of the filter, the delay is a factor of the difference between the current and previous value of $\mathrm{X}(\mathrm{t})$. This provides a varying change in phase with the rate of change of the signal. This characteristic means that the filter will work for a range of frequencies without needing to be reconfigured. Figure 4 shows the effect a fractional delay has on the phase of the signal before entering the SDM, the additional phase added to the system now allows the image cancelation to be preformed fully. The removal of the image allows for full utilization of the bandwidth within the notch of the sigma delta.

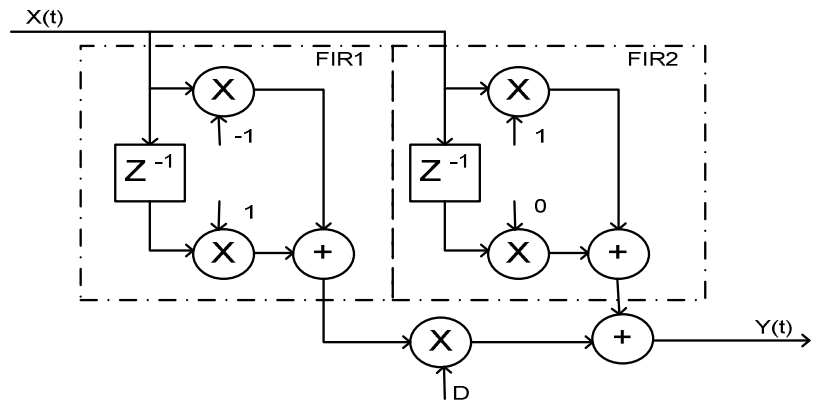

Figure 3 Lagrange Filter using Farrow structure.

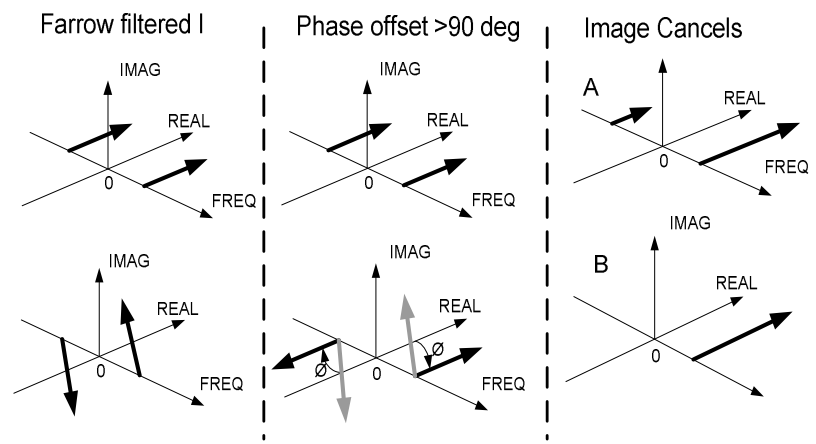

Figure 4 The effects of a fractional delay (A) result before fractional delay filter is added (B) result after fractional delay filter is added.

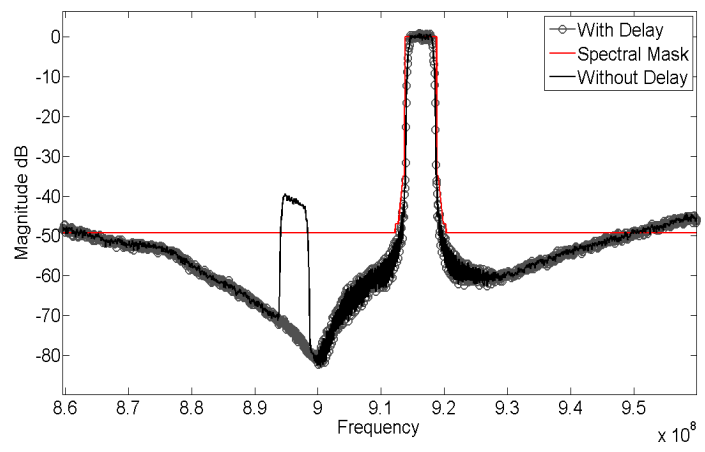

Figure 5 Quadrature mixing, effect of fractional delay
Figure 5 shows the effect the fractional delay has on the output of the system. The image is at $-40 \mathrm{dBc}$, this is above the spectral mask floor for $3 \mathrm{GPP}$ and as such would be required to be removed, however after a relative fractional delay is added between the I and Q signal paths the image is below the NTF of the SDM.

\section{RESULTS}

The simulations are carried out in Matlab using an ideal $2^{\text {nd }}$ order low pass SDM structure and a measured WCDMA signal interpolated to the required sample frequency $(1.8125 \mathrm{GHz})$. Figure 6 shows an example of the image created by Low-IF up conversion without using the quadrature up conversion method. To transmit the signal and still meet spectral emission specifications the image will have to be filtered. In order to do this a filter with extremely high $Q$ is required to implement filtering with high roll-off close to the carrier.

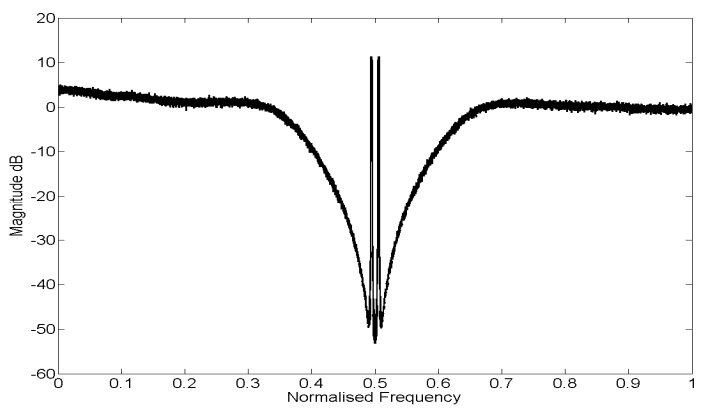

Figure 6 Low Pass SDM up converted to FS/2

The signal pass band in this approach is equivalent to the frequency range in $\mathrm{Hz}$ between the two points at which the NTF rises above $(-49 \mathrm{dBc})$. In the case of the SDM implementation without relative phase correction between I and Q signals an image will be generated and protrude above the spectral mask as in Figure 5. This will reduce the bandwidth of the system, however by introducing a fractional delay in one path relative to the other this image can be further attenuated and the bandwidth increased as in Figure 5.

Quantization noise generated by the SDM will have to be filtered out after transmit signal amplification. Using a commercially available duplexer filter response [6], an inverted filter mask was generated. By plotting the inverse of the filter mask over the output of the sigma delta modulator and assuming that the power amplification stage is linear it can be seen that a commercial duplexer would be capable of removing the out of band quantization noise to meet the performance required for transmission of a WCDMA signal. Figure 7 shows the inverted frequency response of a commercial base station duplexer filter, this shows that the output filter provides the required attenuation to remove the quantization noise. The inverted filter pass band is just below 
the bottom of the WCDMA base station spectral mask requirement [7]. As shown by the difference between the inverted filter mask on top of the SDM spectrum, the out of band attenuation provided by the duplexor is enough to suppress the quantization noise in this case. Therefore the output, after linear amplification and filtering in the duplexer, will meet spectral emission requirements.

In band power vs total power is an important measure of the performance of a SDM. It compares the power of the wanted in band signal with the total signal power including the out of band quantization noise. This is an important factor in the overall efficiency of the power amplifier as the SDM will be driving the switchmode PA stage it will also have to amplify the quantization noise and therefore it will reduce the efficiency of the Class-S amplifier.

Table 1 shows the comparison between two different SDM architectures. The main advantage in using the phase correction quadrature mixing approach is the removal of the signal image close to the transmit frequency. This in turn creates a larger signal pass band, improves the overall coding efficiency which the operating frequency remains constant and reduces the output filter requirements.

Table 1 SDM comparison

$\begin{array}{ccc}\text { SDM } & \text { High Pass } & \text { Quadrature } \\ \mathrm{F}_{\mathrm{SDM}} & 1.8125 \mathrm{GHz} & 1.8125 \mathrm{GHz} \\ \mathrm{F}_{\text {Carrier }} & 916.24 \mathrm{MHz} & 916.24 \mathrm{MHz} \\ \mathrm{F}_{\mathrm{S}} & 1.8125 \mathrm{GHz} & 3.625 \mathrm{GHz} \\ \text { Bandwidth } & 50 \mathrm{MHz} & 80 \mathrm{MHz} \\ \text { Signal power / Total } & 2.3 \% & 4.3 \%\end{array}$

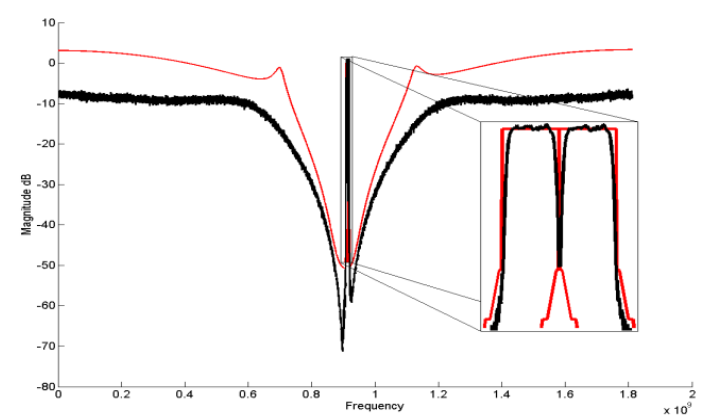

Figure 7 Multicarrier SDM

A major benefit of the phase corrected quadrature mixing approach is the ability to modulate multiple carriers at low IF frequencies. Figure 7 shows the simulation of a multicarrier signal output from a sigma delta modulator within a low bandwidth notch. From this it can be seen that the multi- carrier output can meet spectral mask requirements. A multicarrier signal will however increase the peak power of the signal causing a reduction in dynamic range. With crest factor reduction and careful consideration of the signal modulation scheme used this performance can be improved.

\section{CONCLUSION}

This paper proposes a method of generating a pulse density modulated signal for a Class-S power amplifier. It up converts a signal from a SDM to a transmit frequency without generating an image. This improves the digital signal generation performance used in previous publications, by increasing the modulator signal pass band, improving the modulator coding efficiency and enables interference free multi-carrier signal modulation. Current hardware limitations prevent a practical implementation of the system, however with advances in FPGA hardware and high speed SerDes the design is plausible within the foreseeable future.

\section{ACKNOWLEDGMENT}

This paper is based in part upon works supported by Science Foundation Ireland under grant number 08/CE/I1523. The authors gratefully acknowledge this support.

\section{REFERENCE}

[1] S. C. Li, et al., "New high-efficiency 2.5 V/0.45 W RWDM class-D audio amplifier for portable consumer electronics," Circuits and Systems I: Regular Papers, IEEE Transactions on, vol. 52, pp. 1767-1774, 2005.

[2] S. Ralph and R. Farrell, "Using high pass sigma-delta modulation for Class-S power amplifiers," in Circuit Theory and Design, 2007. ECCTD 2007. 18th European Conference on, 2007, pp. 707-710.

[3] T. Podsiadlik, et al., "Pulse width modulation of multilevel delta-sigma output for class S power amplifier," in Circuit Theory and Design, 2009. ECCTD 2009. European Conference on, 2009, pp. 457-460.

[4] A. K. Ong and B. A. Wooley, "A two-path bandpass \&Sigma;\&Delta; modulator for digital IF extraction at 20 MHz," in Solid-State Circuits Conference, 1997. Digest of Technical Papers. 43rd ISSCC., 1997 IEEE International, 1997, pp. 212-213, 459.

[5] K. Walczak, "Fractional delay FIR filters design with enhanced differential evolution," presented at the World academy of sicence and engineering and Technology 2008.

[6] I. TELEWAVE. (2006, 05/04/11). High power combline duplexer. Available: http://www.telewave.com/pdf/TWDS6003.pdf

[7] Frequentis. (2007, B-AMC interference analysis and spectrum requirements. D4(1.1), 30-31. Available: http://www.eurocontrol.int/communications/gallery/content /public/documents/B_AMC\%20Project\%20Deliverable_D4 V11.pdf 